\title{
New insights into the health priorities and needs of older women
}

\author{
Elizabeth Phelan, James LoGerfo
}

ß See related article page 153

M odern medicine has recognized that paternalism serves patients less well than including them in decision-making regarding their health. In the United States, the Institute of Medicine has called for health care systems that respect patients' values and preferences and provide information that patients need and want. ${ }^{1}$

In this issue, Tannenbaum and colleagues provide several insights regarding what health priorities are of concern to older women and what their perceptions are about the degree to which their priorities are being addressed by their health care providers. ${ }^{2}$ In their report of a survey of women aged 55-95 years across Canada, they found that prevention of memory loss, knowledge of medication side effects and correction of visual impairment were rated as the top health priorities. Nearly as highly ranked were osteoporosis, loss of muscle strength, falls and pain control. At first glance, these priorities seem disparate. On closer examination, however, their shared feature appears to be a choice for health maintenance strategies that sustain or improve health, promote continued independence and enhance quality of life. This is not surprising, given the aging sample studied.

These women's health priorities also reflect areas that tend to be underemphasized in the delivery of routine primary care of older adults. Others have observed that care for geriatric conditions (falls, dementia, urinary incontinence) is less adequate than care for general medical conditions. ${ }^{3}$ Indeed, there appears to be a mismatch between what providers focus on and what the women in Tannenbaum and colleagues' study want, with the vast majority of women reporting that their health care providers more frequently addressed a different set of issues: stroke, heart disease, breast cancer and pneumonia. As a group, vascular disease, cancer and infections reflect a classic disease focus; ${ }^{4}$ emphasis on these diseases in particular is not surprising, since together they represent the main causes of death and disability among older adults in developed countries. It is possible, of course, that women's priorities are driven by what is not addressed in the medical encounter; if stroke, heart disease, breast cancer and pneumonia were in fact neglected on the part of their health care providers, women might have rated these health priorities more highly.
What was the basis for these women's priorities, and why did they give proven interventions such as exercise lower priority? Is this again simply a reflection of the emphasis of primary care as it is currently practised, in the sense that patients do not expect their provider of routine medical care to cover these topics as part of their clinical encounter? Is it a reflection of the fact that this was a fairly well-educated sample, more likely to have fewer chronic conditions and to have already adopted health-promoting habits (e.g., regular exercise) so as to make these areas of lower priority? Further qualitative research into the basis for the women's priority ratings may help to shed light on these provocative but as yet unexplained findings.

Tannenbaum and colleagues observed that women who indicated that particular health issues were of great concern or importance to them were more likely to perceive that the concern was being addressed, compared with women who felt the issues were not of great concern or importance. This finding suggests that patients (and their families) may be their own best advocates in having their health concerns and needs met, and it lends support for the patient-empowering approach that is advocated by the Chronic Care Model. ${ }^{5}$ This model depicts the patient at the heart of the clinical encounter, knowledgeable about his or her conditions, confident and skilled in his or her ability to self-manage, and prepared to participate in partnership with his or her health care provider to optimize care of those conditions. ${ }^{6}$ Efforts to optimize patient participation in the clinical encounter and across care settings should be a high priority for healthcare systems as they seek concrete ways by which to achieve the goals set forth by the US Institute of Medicine while at the same time reduce costs.?

Variations in care are suggested by Tannenbaum and colleagues' study. Variations in preference-sensitive and supply-sensitive care have been documented in the United States. ${ }^{8}$ Although the present study cannot confirm or disprove actual variations in care, or determine the nature of the variation (e.g., variation in care not driven by patient preference, such as the use of $\beta$-blockers after myocardial infarction, or variation in preference-sensitive care or supply-sensitive care), there is strong reason to believe that variation is likely occurring, given the survey's focus on 
preference-driven procedures (e.g., vaccinations, mammography) and the potential for varying quantity and availability of health care resources within Canada's health care system. Insofar as variations are related to patient preference, variation is not necessarily problematic.

How should clinicians incorporate the findings of this study into their practice? First, they should be somewhat cautious in interpreting the results, given the study's limitations (low response rate, sampling bias and recall bias). Second, although women in the study reported that lifestyle factors such as exercise were of low priority to them, clinicians should not be dissuaded from counselling their older patients on these issues. The answer may be to find ways to incorporate discussion of these topics into the context of expressed goals and values of the individual patient. For example, evidence suggests that exercise enhances cognitive performance, ${ }^{9}$ relieves pain, increases muscle strength, improves balance and reduces the risk of chronic diseases (e.g., osteoporosis, hypertension and diabetes) that are associated with functional decline ${ }^{10}$ and can also improve control of chronic conditions if they are already established. If clinicians can be clear with patients about the benefits of interventions such as exercise and situate those benefits within the context of a patient's priorities, patients may be more likely to perceive that their needs and priorities have been understood and attended to.

Health care professionals have much to learn from patients about their health concerns and needs and their perceptions of how well those concerns are being addressed. I hope Tannenbaum and colleagues' study inspires others to pursue further work in this important but understudied area.
From the Division of Gerontology and Geriatric Medicine (Phelan) and the Division of General Internal Medicine (LoGerfo), Department of Medicine, University of Washington, Seattle, Wash.

Competing interests: None declared.

Contributors: Both authors contributed to the writing and revising of the article and approved the final version submitted for publication.

\section{References}

1. Committee on Quality of Healthcare in America; Institute of Medicine. Crossing the quality chasm: a new bealth system for the 21st century. Washington, DC: National Academy Press; 2001.

2. Tannenbaum C, Mayo N, Ducharme F. Older women's health priorities and perceptions of care delivery: results of the WOW health survey. CMAJ 2005;173(2):153-9.

3. Wenger NS, Solomon DH, Roth CP, MacLean CH, Saliba D, Kamberg CJ, et al. The quality of medical care provided to vulnerable community-dwelling older patients. Ann Intern Med 2003;139:740-7.

4. Tinetti ME, Fried T. The end of the disease era. Am 7 Med 2004;116:179-85.

5. Wagner EH. Chronic disease management: what will it take to improve care for chronic illness? Eff Clin Pract 1998;1:2-4.

6. Holman H, Lorig KR. Patients as partners in managing chronic disease. Partnership is a prerequisite for effective and efficient health care. BM7 2000;320:526-7.

7. Lorig KR, Sobel DS, Stewart AL, Brown BW Jr, Bandura A, Ritter P, et al. Evidence suggesting that a chronic disease self-management program can improve health status while reducing hospitalization: a randomized trial. Med Care 1999;37:5-14.

8. Wennberg JE. Unwarranted variations in healthcare delivery: implications for academic medical centres. BM7 2002;325:961-4.

9. Colcombe S, Kramer AF. Fitness effects on the cognitive function of older adults: a meta-analytic study. Psychol Sci 2003;14:125-30.

10. Stuck AE, Walthert JM, Nikolaus T, Bula CJ, Hohmann C, Beck JC. Risk factors for functional status decline in community-living elderly people: a systematic literature review. Soc Sci Med 1999;48:445-69.

Correspondence to: Dr. Elizabeth Phelan, Division of Gerontology and Geriatric Medicine, Department of Medicine, University of Washington, 325-9th Ave., Box 359755,

Seattle WA 98104-2499, USA; fax 206 744-9976;

phelane@u.washington.edu

\section{Clinical trial registration}

CMAJ will consider clinical trials for publication only if they have been registered in a publicly accessible clinical trials registry before the enrolment of the first patient. This policy applies to trials that start recruiting on or after July 1, 2005. For trials that began enrolment before this date, registration is required by Sept. 13, 2005. The criteria for acceptable registration are described in CMAJ (2005;172[13]:1700-2). 